\title{
CONVERGENCE RATES IN THE IMPLICIT RENEWAL THEOREM ON TREES
}

\author{
PREDRAG R. JELENKOVIĆ* ${ }^{*}$ AND \\ MARIANA OLVERA-CRAVIOTO, ${ }^{* *}$ Columbia University
}

\begin{abstract}
We consider possibly nonlinear distributional fixed-point equations on weighted branching trees, which include the well-known linear branching recursion. In Jelenković and Olvera-Cravioto (2012), an implicit renewal theorem was developed that enables the characterization of the power-tail asymptotics of the solutions to many equations that fall into this category. In this paper we complement the analysis in our 2012 paper to provide the corresponding rate of convergence.
\end{abstract}

Keywords: Implicit renewal theory; weighted branching process; branching random walk; multiplicative cascade; rate of convergence; smoothing transform; stochastic recursion; power law; large deviation; stochastic fixed-point equation

2010 Mathematics Subject Classification: Primary 60H25

Secondary 60F10; 60K05; 60J80

\section{Introduction}

Distributional fixed-point equations of the form

$$
R \stackrel{\mathrm{D}}{=} f\left(C_{i}, R_{i}, 1 \leq i \leq N\right)
$$

where $f(\cdot)$ is a possibly random real-valued function, $N \in \mathbb{N} \cup\{\infty\}, \mathbb{N}=\{0,1,2,3, \ldots\}$, $\left\{C_{i}\right\}_{i \in \mathbb{N}}$ are real-valued random weights, and $\left\{R_{i}\right\}_{i \in \mathbb{N}}$ are independent and identically distributed (i.i.d.) copies of $R$, independent of $\left(N, C_{1}, C_{2}, \ldots\right)$, appear in many applications in applied probability, e.g. analysis of algorithms and statistical physics; see [1], [6], [8], [9], [10], and [11] for more details. Throughout the paper, ' $=$ ' stands for equality in distribution.

As previously stated in the abstract, the work in [10] provides an implicit renewal theorem (Theorem 3.4) that enables the characterization of the power-tail behavior of the solution $R$ to (1.1). The results in [10] fully generalize the implicit renewal theorem of Goldie [7], which was derived for equations of the form $R \stackrel{\mathrm{D}}{=} f(C, R)$ (equivalent to $N \equiv 1$ in our case), to recursions (fixed-point equations) on trees. The work in [7], for the $N \equiv 1$ case, also includes the rate of convergence in the implicit renewal theorem. Similarly, in this paper we complement Theorem 3.4 of [10] by deriving its corresponding convergence rate.

More specifically, Theorem 3.4 of [10] provides a general tool to study the tail behavior of the endogenous solutions to fixed-point equations of the type in (1.1), such as the linear and max-plus branching equations; see [9] and [10] for more details. This theorem,

Received 7 November 2012; revision received 1 February 2013.

* Postal address: Department of Electrical Engineering, Columbia University, New York, NY 10027, USA. Email address: predrag@ee.columbia.edu

** Postal address: Department of Industrial Engineering and Operations Research, Columbia University, New York, NY 10027, USA. Email address: molvera@ieor.columbia.edu 
under the natural moment assumption $\mathbb{E}\left[\sum_{i=1}^{N}\left|C_{i}\right|^{\alpha}\right]=1$, with positive derivative at $\alpha, 0<$ $\mathbb{E}\left[\sum_{i=1}^{N}\left|C_{i}\right|^{\alpha} \log \left|C_{i}\right|\right]<\infty$, characterizes both left and right power-law tails of $R$, e.g.

$$
\mathbb{P}(R>t) \sim H_{+} t^{-\alpha} \quad \text { as } t \rightarrow \infty .
$$

This was a natural generalization of the result in [7] for $N \equiv 1$ to the branching case. In this paper, under the expected additional assumption $\mathbb{E}\left[\sum_{i=1}^{N}\left|C_{i}\right|^{\alpha+\theta}\right]<\infty, \theta>0$, and some minor technical conditions, we determine the corresponding rate of convergence, i.e.

$$
\left|t^{\alpha} \mathbb{P}(R>t)-H_{+}\right|=o\left(t^{-\theta}\right) \quad \text { as } t \rightarrow \infty .
$$

Previously, this result was derived in [7] in Theorems 3.2 and 3.3 for $N \equiv 1$. Our extension to trees is facilitated by a matrix form derivation of Corollary 3.4 of [7] that allows the treatment of both nonnegative and real-valued weights simultaneously.

Our main theorem, Theorem 3.1, can be used to determine the rate of convergence of the power-law tails of various solutions to multiplicative max-plus recursions, such as those studied in [9] and [10]. Our recent work is motivated by the nonhomogeneous multiplicative branching recursion

$$
R \stackrel{\mathrm{D}}{=} \sum_{i=1}^{N} C_{i} R_{i}+Q,
$$

where $N \in \mathbb{N} \cup\{\infty\},\left\{C_{i}\right\}_{i \in \mathbb{N}}$ are real-valued random weights, $Q$ is a nonzero real-valued random variable, and $\left\{R_{i}\right\}_{i \in \mathbb{N}}$ are i.i.d. copies of $R$, independent of $\left(Q, N, C_{1}, C_{2}, \ldots\right)$. In the context of deterministic weights this recursion was previously studied in [3]; more recently, the work in [2] characterizes the additional nonendogenous solutions. Historically, the homogeneous version of this equation, $Q \equiv 0$, has been extensively studied in the literature; for recent work, see [4] and the references therein.

\section{Weighted branching tree}

We use the model from [10] for defining a weighted branching tree. First we construct a random tree $\mathcal{T}$. We use the notation $\varnothing$ to denote the root node of $\mathcal{T}$, and $A_{n}, n \geq 0$, to denote the set of all individuals in the $n$th generation of $\mathcal{T}, A_{0}=\{\varnothing\}$. Let $Z_{n}$ be the number of individuals in the $n$th generation, that is, $Z_{n}=\left|A_{n}\right|$, where $|\cdot|$ denotes the cardinality of a set; in particular, $Z_{0}=1$.

Next, let $\mathbb{N}_{+}=\{1,2,3, \ldots\}$ be the set of positive integers and let $U=\bigcup_{k=0}^{\infty}\left(\mathbb{N}_{+}\right)^{k}$ be the set of all finite sequences $\boldsymbol{i}=\left(i_{1}, i_{2}, \ldots, i_{n}\right) \in U$, where by convention $\mathbb{N}_{+}^{0}=\{\varnothing\}$ contains the null sequence $\varnothing$. To ease the exposition, for a sequence $\boldsymbol{i}=\left(i_{1}, i_{2}, \ldots, i_{k}\right) \in U$, we write $\boldsymbol{i} \mid n=\left(i_{1}, i_{2}, \ldots, i_{n}\right)$, provided $k \geq n$, and $\boldsymbol{i} \mid 0=\varnothing$ to denote the index truncation at level $n, n \geq 0$. Also, for $i \in A_{1}$, we simply use the notation $i=i_{1}$, that is, without the parenthesis. Similarly, for $\boldsymbol{i}=\left(i_{1}, \ldots, i_{n}\right)$, we will use $(\boldsymbol{i}, j)=\left(i_{1}, \ldots, i_{n}, j\right)$ to denote the index concatenation operation; if $i=\varnothing$ then $(i, j)=j$.

We iteratively construct the tree as follows. Let $N$ be the number of individuals born to the root node $\varnothing, N_{\varnothing}=N$, and let $\left\{N_{i}\right\}_{i \in U, i \neq \varnothing}$ be i.i.d. copies of $N$. Now define

$$
A_{1}=\{i \in \mathbb{N}: 1 \leq i \leq N\}, \quad A_{n}=\left\{\left(\boldsymbol{i}, i_{n}\right) \in U: i \in A_{n-1}, 1 \leq i_{n} \leq N_{i}\right\} .
$$

It follows that the number of individuals $Z_{n}=\left|A_{n}\right|$ in the $n$th generation, $n \geq 1$, satisfies the branching recursion

$$
Z_{n}=\sum_{i \in A_{n-1}} N_{i}
$$




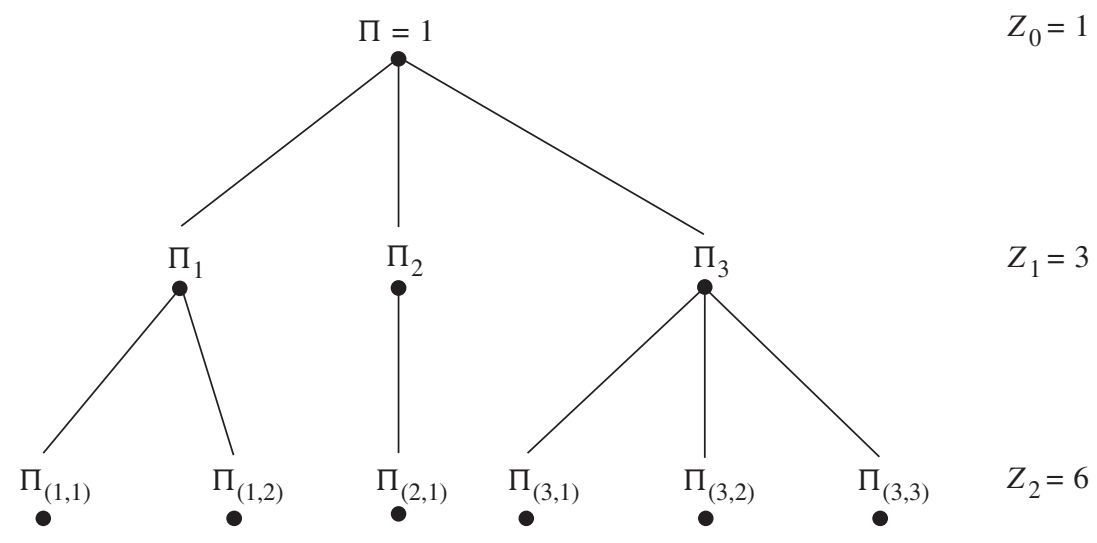

FIGURE 1: Weighted branching tree.

Now, we construct the weighted branching tree $\mathcal{T}_{C}$ as follows. Let

$$
\left\{\left(N_{i}, C_{(i, 1)}, C_{(i, 2)}, \ldots\right)\right\}_{i \in U, i \neq \varnothing}
$$

be a sequence of i.i.d. copies of $\left(N, C_{1}, C_{2}, \ldots\right)$. Here $N_{\varnothing}$ determines the number of nodes in the first generation of $\mathcal{T}$ according to (2.1), and each node in the first generation is then assigned its corresponding vector $\left(N_{i}, C_{(i, 1)}, C_{(i, 2)}, \ldots\right)$ from the i.i.d. sequence defined above. In general, for $n \geq 2$, to each node $i \in A_{n-1}$ we assign its corresponding $\left(N_{i}, C_{(i, 1)}, C_{(i, 2)}, \ldots\right)$ from the sequence and construct $A_{n}=\left\{\left(i, i_{n}\right) \in U: i \in A_{n-1}, 1 \leq i_{n} \leq N_{i}\right\}$. For each node in $\mathcal{T}_{C}$, we also define the weight $\Pi_{\left(i_{1}, \ldots, i_{n}\right)}$ via the recursion

$$
\Pi_{i_{1}}=C_{i_{1}}, \quad \Pi_{\left(i_{1}, \ldots, i_{n}\right)}=C_{\left(i_{1}, \ldots, i_{n}\right)} \Pi_{\left(i_{1}, \ldots, i_{n-1}\right)}, \quad n \geq 2,
$$

where $\Pi=1$ is the weight of the root node. Note that the weight $\Pi_{\left(i_{1}, \ldots, i_{n}\right)}$ is equal to the product of all the weights $C_{(\cdot)}$ along the branch leading to node $\left(i_{1}, \ldots, i_{n}\right)$, as depicted in Figure 1.

\section{Rate of convergence in the implicit renewal theorem on trees}

In this section we present an extension of Corollary 3.4 of [7]. Similarly as in [10], the key observation that facilitates this generalization is the following lemma which shows that a certain measure on a tree is a matrix product measure; its proof can be found in [10]. For the case of positive weights, a similar observation was made for a scalar measure in [5]. Throughout the paper, we use the standard convention $0^{\alpha} \log 0=0$ for all $\alpha>0$.

Let $\boldsymbol{F}=\left(F_{i j}\right)$ be an $n \times n$ matrix whose elements are finite measures on $\mathbb{R}$. The convolution $\boldsymbol{F} * \boldsymbol{G}$ of two such matrices is the matrix with elements $(\boldsymbol{F} * \boldsymbol{G})_{i j} \triangleq \sum_{k=1}^{n} F_{i k} * G_{k j}, i, j=$ $1, \ldots, n$, where $F_{i k} * G_{k j}$ is the convolution of individual measures; ' $\triangleq$ ' stands for equal by definition.

Definition 3.1. A matrix renewal measure is the matrix of measures

$$
\mathbf{U}=\sum_{k=0}^{\infty} \boldsymbol{F}^{* k},
$$

where $\boldsymbol{F}^{* 1}=\boldsymbol{F}, \boldsymbol{F}^{*(k+1)}=\boldsymbol{F}^{* k} * \boldsymbol{F}=\boldsymbol{F} * \boldsymbol{F}^{* k}, \boldsymbol{F}^{* 0}=\delta_{0} \mathbf{I}, \delta_{0}$ is the point measure at 0 , and I is the $n \times n$ identity matrix. 
Lemma 3.1. Let $\mathcal{T}_{C}$ be the weighted branching tree defined by the vector $\left(N, C_{1}, C_{2}, \ldots\right)$, where $N \in \mathbb{N} \cup\{\infty\}$ and the $C_{i}$ s are real valued. For any $n \in \mathbb{N}$ and $i \in A_{n}$, let $V_{i}=\log \left|\Pi_{i}\right|$ and $X_{i}=\operatorname{sgn}\left(\Pi_{i}\right) ; V_{\varnothing} \equiv 0, X_{\varnothing} \equiv 1$. For $\alpha>0$, define the measures

$$
\begin{aligned}
& \mu_{n}^{(+)}(\mathrm{d} t)=\mathrm{e}^{\alpha t} \mathbb{E}\left[\sum_{i \in A_{n}} \mathbf{1}\left(X_{i}=1, V_{i} \in \mathrm{d} t\right)\right], \\
& \mu_{n}^{(-)}(\mathrm{d} t)=\mathrm{e}^{\alpha t} \mathbb{E}\left[\sum_{i \in A_{n}} \mathbf{1}\left(X_{i}=-1, V_{i} \in \mathrm{d} t\right)\right],
\end{aligned}
$$

for $n=0,1,2, \ldots$, and let $\eta_{ \pm}(\mathrm{d} t)=\mu_{1}^{( \pm)}(\mathrm{d} t)$. Suppose that $\mathbb{E}\left[\sum_{i=1}^{N}\left|C_{i}\right|^{\alpha} \log \left|C_{i}\right|\right] \geq 0$ and $\mathbb{E}\left[\sum_{i=1}^{N}\left|C_{i}\right|^{\alpha}\right]=1$. Then $\left(\eta_{+}+\eta_{-}\right)(\cdot)$ is a probability measure on $\mathbb{R}$ that places no mass at $-\infty$ and has mean

$$
\int_{-\infty}^{\infty} u \eta_{+}(\mathrm{d} u)+\int_{-\infty}^{\infty} u \eta_{-}(\mathrm{d} u)=\mathbb{E}\left[\sum_{j=1}^{N}\left|C_{j}\right|^{\alpha} \log \left|C_{j}\right|\right] .
$$

Furthermore, if we let $\boldsymbol{\mu}_{n}=\left(\mu_{n}^{(+)}, \mu_{n}^{(-)}\right), \mathbf{e}=(1,0)$, and $\boldsymbol{F}=\left(\begin{array}{ll}\eta_{+} & \eta_{-} \\ \eta_{-} & \eta_{+}\end{array}\right)$, then

$$
\boldsymbol{\mu}_{n}=\left(\mu_{n}^{(+)}, \mu_{n}^{(-)}\right)=(1,0)\left(\begin{array}{ll}
\eta_{+} & \eta_{-} \\
\eta_{-} & \eta_{+}
\end{array}\right)^{* n}=\mathbf{e} \boldsymbol{F}^{* n},
$$

where $\boldsymbol{F}^{* n}$ denotes the nth matrix convolution of $\boldsymbol{F}$ with itself.

In what follows, $\widehat{v}(s)=\int_{-\infty}^{\infty} \mathrm{e}^{s x} v(\mathrm{~d} s)$ denotes the Laplace transform of measure $v$. If $\boldsymbol{F}$ is a matrix of measures then $\widehat{\boldsymbol{F}}(s)$ is the corresponding matrix of Laplace transforms.

Assumption 3.1. Suppose that the matrix of measures

$$
\boldsymbol{F}=\left(\begin{array}{ll}
\eta_{+} & \eta_{-} \\
\eta_{-} & \eta_{+}
\end{array}\right)
$$

satisfies the following. For some $\theta>0$, the equation

$$
\left(1-\widehat{\eta}_{+}(s)\right)^{2}-\left(\widehat{\eta}_{-}(s)\right)^{2}=0
$$

has no roots different from 0 on the strip $\{s \in \mathbb{C}: 0 \leq \mathcal{R}(s) \leq \theta\}$, and there exists an integer $m \geq 1$ such that the Laplace transform of the singular part of $\boldsymbol{F}^{* m}$, denoted by $\widehat{\boldsymbol{F}}_{s}^{* m}(\vartheta)$, has spectral radius strictly smaller than 1 for $\vartheta \in\{0, \theta\}$.

Note that in the following theorem the random variable $R$ and the vector $\left(N, C_{1}, C_{2}, \ldots\right)$ are arbitrary, except for the independence assumption, and, therefore, this result can be applied to any random variable $R$ satisfying the theorem's conditions regardless of whether it satisfies a particular recursion or not.

Theorem 3.1. Let $\left(N, C_{1}, C_{2}, \ldots\right)$ be a random vector, where $N \in \mathbb{N} \cup\{\infty\}$ and the $C_{i}$ s are real valued. Suppose that $\boldsymbol{F}$ satisfies Assumption 3.1 for some $\theta>0$. Furthermore, assume that

$$
\mu \triangleq \mathbb{E}\left[\sum_{j=1}^{N}\left|C_{j}\right|^{\alpha} \log \left|C_{j}\right|\right]>0, \quad \mathbb{E}\left[\sum_{j=1}^{N}\left|C_{j}\right|^{\alpha}\right]=1,
$$




$$
\mathbb{E}\left[\sum_{j=1}^{N}\left|C_{j}\right|^{\alpha}\left(\log \left|C_{j}\right|\right)^{2}\right]<\infty, \text { and } \mathbb{E}\left[\sum_{j=1}^{N}\left|C_{j}\right|^{\gamma}\right]<\infty
$$

for some $0 \leq \gamma<\alpha$, and that $R$ is independent of $\left(N, C_{1}, C_{2}, \ldots\right)$.

1. If $\left\{C_{i}\right\} \geq 0$ almost surely, $\mathbb{E}\left[\left(R^{+}\right)^{\beta}\right]<\infty$ for any $0<\beta<\alpha$, and, for $\sigma \in\{0, \theta\}$,

$$
\int_{0}^{\infty}\left|\mathbb{P}(R>t)-\mathbb{E}\left[\sum_{j=1}^{N} \mathbf{1}\left(C_{j} R>t\right)\right]\right| t^{\alpha+\sigma-1} \mathrm{~d} t<\infty,
$$

or, respectively, $\mathbb{E}\left[\left(R^{-}\right)^{\beta}\right]<\infty$ for any $0<\beta<\alpha$, and, for $\sigma \in\{0, \theta\}$,

$$
\int_{0}^{\infty}\left|\mathbb{P}(R<-t)-\mathbb{E}\left[\sum_{j=1}^{N} \mathbf{1}\left(C_{j} R<-t\right)\right]\right| t^{\alpha+\sigma-1} \mathrm{~d} t<\infty,
$$

then

$$
\left|t^{\alpha} \mathbb{P}(R>t)-H_{+}\right|=o\left(t^{-\theta}\right) \quad \text { as } t \rightarrow \infty,
$$

or, respectively,

$$
\left|t^{\alpha} \mathbb{P}(R<-t)-H_{-}\right|=o\left(t^{-\theta}\right) \quad \text { as } t \rightarrow \infty,
$$

where $0 \leq H_{ \pm}<\infty$ are given by

$$
H_{ \pm}=\frac{1}{\mu} \int_{0}^{\infty} x^{\alpha-1}\left(\mathbb{P}(( \pm 1) R>x)-\mathbb{E}\left[\sum_{j=1}^{N} \mathbf{1}\left(( \pm 1) C_{j} R>x\right)\right]\right) \mathrm{d} x .
$$

2. If $\mathbb{P}\left(C_{j}<0\right)>0$ for some $j \geq 1, \mathbb{E}\left[|R|^{\beta}\right]<\infty$ for any $0<\beta<\alpha$, and both (3.1) and (3.2) are satisfied, then

$$
\left|t^{\alpha} \mathbb{P}(R>t)-H\right|=o\left(t^{-\theta}\right) \quad \text { and } \quad\left|t^{\alpha} \mathbb{P}(R<-t)-H\right|=o\left(t^{-\theta}\right) \quad \text { as } t \rightarrow \infty,
$$

where $0 \leq H=\left(H_{+}+H_{-}\right) / 2<\infty$ is given by

$$
H=\frac{1}{2 \mu} \int_{0}^{\infty} x^{\alpha-1}\left(\mathbb{P}(|R|>x)-\mathbb{E}\left[\sum_{j=1}^{N} \mathbf{1}\left(\left|C_{j} R\right|>x\right)\right]\right) \mathrm{d} x .
$$

Remark 3.1. Note that, when $N \equiv 1$, then (3.1) and (3.2) need to only hold for $\sigma=\theta$, since in this case

$$
\begin{aligned}
\int_{0}^{\infty} & |\mathbb{P}( \pm R>t)-\mathbb{P}( \pm C R>t)| t^{\alpha-1} \mathrm{~d} t \\
& \leq \int_{0}^{1} t^{\alpha-1} \mathrm{~d} t+\int_{1}^{\infty}|\mathbb{P}( \pm R>t)-\mathbb{P}( \pm C R>t)| t^{\alpha+\theta-1} \mathrm{~d} t \\
& <\infty
\end{aligned}
$$

which is equivalent to conditions (3.7) and (3.9) in Theorems 3.2 and 3.3 of [7]. Furthermore, for $N \equiv 1$, our condition $\mathbb{E}\left[|C|^{\alpha}(\log |C|)^{2}\right]<\infty$ is weaker than $\mathbb{E}\left[|C|^{\alpha+\theta}\right]<\infty$ in [7]. However, it is likely that in applications of this theorem to specific recursions one might need to assume that $\mathbb{E}\left[\sum_{1=1}^{N}\left|C_{i}\right|^{\alpha+\theta}\right]<\infty$ in order to verify conditions (3.1) and (3.2). 
Lemma 3.2. Let $\alpha, b>0$ and $0 \leq H<\infty$. Suppose that, for some $\varepsilon>0$,

$$
\left|t^{-b} \int_{0}^{t} b x^{\alpha+b-1} \mathbb{P}(R>x) \mathrm{d} x-H\right|=o\left(t^{-\varepsilon}\right) \text { as } t \rightarrow \infty .
$$

Then

$$
\left|t^{\alpha} \mathbb{P}(R>t)-H\right|=o\left(t^{-\varepsilon}\right) \quad \text { as } t \rightarrow \infty .
$$

Proof. Fix $\delta \in\left(0, \frac{1}{2}\right)$ and note that, as $t \rightarrow \infty$,

$$
\begin{aligned}
\mathbb{P}(R> & t) b t^{\alpha+b} \frac{(1+\delta)^{\alpha+b}-1}{\alpha+b} \\
\geq & \int_{t}^{(1+\delta) t} b x^{\alpha+b-1} \mathbb{P}(R>x) \mathrm{d} x \\
= & ((1+\delta) t)^{b}\left(((1+\delta) t)^{-b} \int_{0}^{(1+\delta) t} b x^{\alpha+b-1} \mathbb{P}(R>x) \mathrm{d} x-H\right) \\
& -t^{b}\left(t^{-b} \int_{0}^{t} b x^{\alpha+b-1} \mathbb{P}(R>x) \mathrm{d} x-H\right)+H((1+\delta) t)^{b}-H t^{b} \\
= & H t^{b}\left((1+\delta)^{b}-1\right)+o\left(t^{b-\varepsilon}\right) .
\end{aligned}
$$

Similarly,

$$
\begin{aligned}
\mathbb{P}(R> & t) b t^{\alpha+b} \frac{1-(1-\delta)^{\alpha+b}}{\alpha+b} \\
\leq & \int_{(1-\delta) t}^{t} b x^{\alpha+b-1} \mathbb{P}(R>x) \mathrm{d} x \\
= & -((1-\delta) t)^{b}\left(((1-\delta) t)^{-b} \int_{0}^{(1-\delta) t} b x^{\alpha+b-1} \mathbb{P}(R>x) \mathrm{d} x-H\right) \\
& +t^{b}\left(t^{-b} \int_{0}^{t} b x^{\alpha+b-1} \mathbb{P}(R>x) \mathrm{d} x-H\right)-H((1-\delta) t)^{b}+H t^{b} \\
= & H t^{b}\left(1-(1-\delta)^{b}\right)+o\left(t^{b-\varepsilon}\right) .
\end{aligned}
$$

Hence, it follows that

$$
\begin{aligned}
H\left(\frac{(\alpha+b)\left((1+\delta)^{b}-1\right)}{b\left((1+\delta)^{\alpha+b}-1\right)}-1\right)+o\left(t^{-\varepsilon}\right) & \leq \mathbb{P}(R>t) t^{\alpha}-H \\
& \leq H\left(\frac{(\alpha+b)\left(1-(1-\delta)^{b}\right)}{b\left(1-(1-\delta)^{\alpha+b}\right)}-1\right)+o\left(t^{-\varepsilon}\right)
\end{aligned}
$$

Now choose $\delta=t^{-2 \varepsilon}$ and use the fact that $(1 \pm \delta)^{c}=1 \pm c \delta+O\left(\delta^{2}\right)$ as $\delta \rightarrow 0$, to obtain $\frac{(\alpha+b)\left((1 \pm \delta)^{b}-1\right)}{b\left((1 \pm \delta)^{\alpha+b}-1\right)}-1=\frac{(\alpha+b)\left(b \delta+O\left(\delta^{2}\right)\right)}{b\left((\alpha+b) \delta+O\left(\delta^{2}\right)\right)}-1=\frac{1+O(\delta)}{1+O(\delta)}-1=O(\delta)=o\left(t^{-\varepsilon}\right)$ as $t \rightarrow \infty$. 
Proof of Theorem 3.1. Define the measures $\eta_{+}$and $\eta_{-}$according to Lemma 3.1, and let

$$
\begin{gathered}
g_{+}(t)=\mathrm{e}^{\alpha t}\left(\mathbb{P}\left(R>\mathrm{e}^{t}\right)-\mathbb{E}\left[\sum_{j=1}^{N} \mathbf{1}\left(C_{j} R>\mathrm{e}^{t}\right)\right]\right), \\
g_{-}(t)=\mathrm{e}^{\alpha t}\left(\mathbb{P}\left(R<-\mathrm{e}^{t}\right)-\mathbb{E}\left[\sum_{j=1}^{N} \mathbf{1}\left(C_{j} R<-\mathrm{e}^{t}\right)\right]\right), \\
\text { and } r(t)=\mathrm{e}^{\alpha t} \mathbb{P}\left(R>\mathrm{e}^{t}\right) .
\end{gathered}
$$

Fix $b>\theta>0$, and define, for any integrable function $f$, the operator

$$
\breve{f}(t)=\int_{-\infty}^{t} b \mathrm{e}^{-b(t-u)} f(u) \mathrm{d} u .
$$

Now, the same arguments used in the proof of Theorem 3.4 of [10] lead to

$$
\breve{r}(t)=\mathbf{e}(\mathbf{U} * \breve{g})(t),
$$

where

$$
\mathbf{e}=(1,0), \quad \breve{\boldsymbol{g}}=\left(\breve{g}_{+}, \breve{g}_{-}\right)^{\top}, \quad \mathbf{U}=\sum_{k=0}^{\infty} \boldsymbol{F}^{* k}, \quad \text { and } \quad \boldsymbol{F}=\left(\begin{array}{ll}
\eta_{+} & \eta_{-} \\
\eta_{-} & \eta_{+}
\end{array}\right) .
$$

Next, we proceed to verify the assumptions of Theorem 2 of [12].

Define $\varphi(t)=\mathrm{e}^{\theta t^{+}}$, and note that

$$
r_{1} \triangleq \lim _{t \rightarrow-\infty} \frac{\log \varphi(t)}{t}=0 \quad \text { and } \quad r_{2} \triangleq \lim _{t \rightarrow \infty} \frac{\log \varphi(t)}{t}=\theta
$$

We will now show that provided (3.1) and (3.2) hold, $\breve{\boldsymbol{g}}$ satisfies the following properties:

(a) $\breve{\mathrm{g}} \in L_{1}(\mathbb{R})$;

(b) $\breve{\boldsymbol{g}}(t) \varphi(t) \in L_{\infty}(\mathbb{R})$;

(c) $\breve{\boldsymbol{g}}(t) \varphi(t) \rightarrow 0$ as $|t| \rightarrow \infty$ outside of a set of Lebesgue measure 0 ;

(d) $\varphi(t) \int_{t}^{\infty}|\breve{\boldsymbol{g}}(x)| \mathrm{d} x \rightarrow 0$ as $t \rightarrow \infty$ and $\varphi(t) \int_{-\infty}^{t}|\breve{\boldsymbol{g}}(x)| \mathrm{d} x \rightarrow 0$ as $t \rightarrow-\infty$.

For part (a), note that, by (3.1) and (3.2), we know that $g_{ \pm} \in L_{1}(\mathbb{R})$, so, by Lemma 9.2 of [7], $\breve{g}_{ \pm}$is directly Riemann integrable, and, in particular, $\breve{\boldsymbol{g}} \in L_{1}(\mathbb{R})$.

For part (b), note that

$$
\begin{aligned}
\breve{g}_{ \pm}(t) \varphi(t) & =b \mathrm{e}^{-b t+\theta t^{+}} \int_{-\infty}^{t} \mathrm{e}^{b u} g_{ \pm}(u) \mathrm{d} u \\
& =b \mathrm{e}^{-b t+\theta t^{+}} \int_{-\infty}^{t} \mathrm{e}^{(b+\alpha) u}\left(\mathbb{P}\left(( \pm 1) R>\mathrm{e}^{u}\right)-\mathbb{E}\left[\sum_{j=1}^{N} \mathbf{1}\left(( \pm 1) C_{j} R>\mathrm{e}^{u}\right)\right]\right) \mathrm{d} u \\
& =b \mathrm{e}^{-b t+\theta t^{+}} \int_{0}^{\mathrm{e}^{t}} x^{b+\alpha-1}\left(\mathbb{P}(( \pm 1) R>x)-\mathbb{E}\left[\sum_{j=1}^{N} \mathbf{1}\left(( \pm 1) C_{j} R>x\right)\right]\right) \mathrm{d} x .
\end{aligned}
$$


Since, for $0 \leq x \leq \mathrm{e}^{t}$, we have $x^{b} \leq \mathrm{e}^{(b-\theta) t} x^{\theta}$, it follows that

$$
\begin{aligned}
\sup _{t \geq 0}\left|\breve{g}_{ \pm}(t) \varphi(t)\right| & \leq \sup _{t \geq 0} b \int_{0}^{\mathrm{e}^{t}} x^{\alpha+\theta-1}\left|\mathbb{P}(( \pm 1) R>x)-\mathbb{E}\left[\sum_{j=1}^{N} \mathbf{1}\left(( \pm 1) C_{j} R>x\right)\right]\right| \mathrm{d} x \\
& <\infty,
\end{aligned}
$$

by (3.1) and (3.2). For the supremum over the negative reals, note that, since $0 \leq x \leq \mathrm{e}^{t}$, we have $x^{b} \leq \mathrm{e}^{b t}$; hence,

$$
\begin{aligned}
\sup _{t<0}\left|\breve{g}_{ \pm}(t) \varphi(t)\right| & \leq \sup _{t<0} b \int_{0}^{\mathrm{e}^{t}} x^{\alpha-1}\left|\mathbb{P}(( \pm 1) R>x)-\mathbb{E}\left[\sum_{j=1}^{N} \mathbf{1}\left(( \pm 1) C_{j} R>x\right)\right]\right| \mathrm{d} x \\
& <\infty .
\end{aligned}
$$

To verify (c) for $t \rightarrow \infty$, note that if

$$
\int_{0}^{\infty} x^{b+\alpha-1}\left|\mathbb{P}(( \pm 1) R>x)-\mathbb{E}\left[\sum_{j=1}^{N} \mathbf{1}\left(( \pm 1) C_{j} R>x\right)\right]\right| \mathrm{d} x<\infty
$$

then we trivially have $\lim _{t \rightarrow \infty} \breve{g}_{ \pm}(t) \varphi(t)=0$; if it is infinite, we can apply l'Hôpital's rule to obtain

$$
\begin{aligned}
\lim _{t \rightarrow \infty} \breve{g}_{ \pm}(t) \varphi(t) & \leq \lim _{t \rightarrow \infty} \frac{b \mathrm{e}^{(b+\alpha-1) t}\left|\mathbb{P}\left(( \pm 1) R>\mathrm{e}^{t}\right)-\mathbb{E}\left[\sum_{j=1}^{N} \mathbf{1}\left(( \pm 1) C_{j} R>\mathrm{e}^{t}\right)\right]\right| \mathrm{e}^{t}}{(b-\theta) \mathrm{e}^{(b-\theta) t}} \\
& =\frac{b}{b-\theta} \lim _{t \rightarrow \infty} \mathrm{e}^{(\alpha+\theta) t}\left|\mathbb{P}\left(( \pm 1) R>\mathrm{e}^{t}\right)-\mathbb{E}\left[\sum_{j=1}^{N} \mathbf{1}\left(( \pm 1) C_{j} R>\mathrm{e}^{t}\right)\right]\right|
\end{aligned}
$$

which is 0 by (3.1) and (3.2). The fact that $\lim _{t \rightarrow-\infty} \breve{g}_{ \pm}(t) \varphi(t)=0$ follows from the estimates used to verify (b).

For part (d), note that, for $t \geq 0$,

$$
\begin{aligned}
\varphi(t) & \int_{t}^{\infty}\left|\breve{g}_{ \pm}(x)\right| \mathrm{d} x \\
& =\mathrm{e}^{\theta t} \int_{t}^{\infty}\left|b \mathrm{e}^{-b x} \int_{-\infty}^{x} \mathrm{e}^{(b+\alpha) u}\left(\mathbb{P}\left(( \pm 1) R>\mathrm{e}^{u}\right)-\mathbb{E}\left[\sum_{j=1}^{N} \mathbf{1}\left(( \pm 1) C_{j} R>\mathrm{e}^{u}\right)\right]\right) \mathrm{d} u\right| \mathrm{d} x \\
& \leq b \mathrm{e}^{\theta t} \int_{t}^{\infty} \mathrm{e}^{-b x} \int_{-\infty}^{x} \mathrm{e}^{(b+\alpha) u}\left|\mathbb{P}\left(( \pm 1) R>\mathrm{e}^{u}\right)-\mathbb{E}\left[\sum_{j=1}^{N} \mathbf{1}\left(( \pm 1) C_{j} R>\mathrm{e}^{u}\right)\right]\right| \mathrm{d} u \mathrm{~d} x \\
& =b \mathrm{e}^{\theta t} \int_{-\infty}^{\infty} \int_{t \vee u}^{\infty} \mathrm{e}^{-b x} \mathrm{e}^{(b+\alpha) u}\left|\mathbb{P}\left(( \pm 1) R>\mathrm{e}^{u}\right)-\mathbb{E}\left[\sum_{j=1}^{N} \mathbf{1}\left(( \pm 1) C_{j} R>\mathrm{e}^{u}\right)\right]\right| \mathrm{d} x \mathrm{~d} u \\
& =\mathrm{e}^{\theta t} \int_{-\infty}^{\infty} \mathrm{e}^{-b(t \vee u)} \mathrm{e}^{(b+\alpha) u}\left|\mathbb{P}\left(( \pm 1) R>\mathrm{e}^{u}\right)-\mathbb{E}\left[\sum_{j=1}^{N} \mathbf{1}\left(( \pm 1) C_{j} R>\mathrm{e}^{u}\right)\right]\right| \mathrm{d} u \\
& =\mathrm{e}^{-(b-\theta) t} \int_{0}^{\mathrm{e}^{t}} v^{b+\alpha-1}\left|\mathbb{P}(( \pm 1) R>v)-\mathbb{E}\left[\sum_{j=1}^{N} \mathbf{1}\left(( \pm 1) C_{j} R>v\right)\right]\right| \mathrm{d} v
\end{aligned}
$$




$$
\begin{aligned}
& +\mathrm{e}^{\theta t} \int_{\mathrm{e}^{t}}^{\infty} v^{\alpha-1}\left|\mathbb{P}(( \pm 1) R>v)-\mathbb{E}\left[\sum_{j=1}^{N} \mathbf{1}\left(( \pm 1) C_{j} R>v\right)\right]\right| \mathrm{d} v \\
\leq & \mathrm{e}^{-(b-\theta) t} \int_{0}^{\mathrm{e}^{t / 2}} v^{b+\alpha-1}\left|\mathbb{P}(( \pm 1) R>v)-\mathbb{E}\left[\sum_{j=1}^{N} \mathbf{1}\left(( \pm 1) C_{j} R>v\right)\right]\right| \mathrm{d} v \\
& +\int_{\mathrm{e}^{t / 2}}^{\infty} v^{\alpha+\theta-1}\left|\mathbb{P}(( \pm 1) R>v)-\mathbb{E}\left[\sum_{j=1}^{N} \mathbf{1}\left(( \pm 1) C_{j} R>v\right)\right]\right| \mathrm{d} v,
\end{aligned}
$$

where in the last inequality we split the range of integration of the first integral into $\left[0, \mathrm{e}^{t / 2}\right]$ and $\left[\mathrm{e}^{t / 2}, \mathrm{e}^{t}\right]$, and used the inequalities $v^{b} \leq \mathrm{e}^{(b-\theta) t} v^{\theta}$ for $\mathrm{e}^{t / 2} \leq v \leq \mathrm{e}^{t}$ and $\mathrm{e}^{\theta t} \leq v^{\theta}$ for $v \geq \mathrm{e}^{t}$. The integral in (3.5) converges to 0 as $t \rightarrow \infty$ since it is the tail of a finite integral; the integral in (3.4) is bounded by

$$
\mathrm{e}^{-(b-\theta) t / 2} \int_{0}^{\infty} v^{\alpha+\theta-1}\left|\mathbb{P}(( \pm 1) R>v)-\mathbb{E}\left[\sum_{j=1}^{N} \mathbf{1}\left(( \pm 1) C_{j} R>v\right)\right]\right| \mathrm{d} v
$$

which also converges to 0 as $t \rightarrow \infty$. Similarly, for $t<0$,

$$
\begin{aligned}
\varphi(t) & \int_{-\infty}^{t}\left|\breve{g}_{ \pm}(x)\right| \mathrm{d} x \\
& \leq b \int_{-\infty}^{t} \mathrm{e}^{-b x} \int_{-\infty}^{x} \mathrm{e}^{(b+\alpha) u}\left|\mathbb{P}\left(( \pm 1) R>\mathrm{e}^{u}\right)-\mathbb{E}\left[\sum_{j=1}^{N} \mathbf{1}\left(( \pm 1) C_{j} R>\mathrm{e}^{u}\right)\right]\right| \mathrm{d} u \mathrm{~d} x \\
& =\int_{-\infty}^{t} \mathrm{e}^{(b+\alpha) u}\left|\mathbb{P}\left(( \pm 1) R>\mathrm{e}^{u}\right)-\mathbb{E}\left[\sum_{j=1}^{N} \mathbf{1}\left(( \pm 1) C_{j} R>\mathrm{e}^{u}\right)\right]\right|\left(\mathrm{e}^{-b u}-\mathrm{e}^{-b t}\right) \mathrm{d} u \\
& =\int_{0}^{\mathrm{e}^{t}} v^{b+\alpha-1}\left(v^{-b}-\mathrm{e}^{-b t}\right)\left|\mathbb{P}(( \pm 1) R>v)-\mathbb{E}\left[\sum_{j=1}^{N} \mathbf{1}\left(( \pm 1) C_{j} R>v\right)\right]\right| \mathrm{d} v \\
& \leq \int_{0}^{\mathrm{e}^{t}} v^{\alpha-1}\left|\mathbb{P}(( \pm 1) R>v)-\mathbb{E}\left[\sum_{j=1}^{N} \mathbf{1}\left(( \pm 1) C_{j} R>v\right)\right]\right| \mathrm{d} v \\
& \rightarrow 0 \text { as } t \rightarrow-\infty .
\end{aligned}
$$

We split the rest of the proof into two different cases.

Case 1: $C_{i} \geq 0$ for all $i$. For this case, we have $\eta_{-} \equiv 0$, from where it follows that

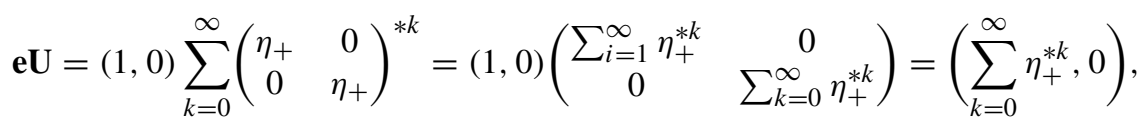

which in turn implies that

$$
\breve{r}(t)=\sum_{k=0}^{\infty}\left(\breve{g}_{+} * \eta_{+}^{* k}\right)(t)
$$


We can then think of this case as a standard one-dimensional problem by renaming $\boldsymbol{F}=\eta_{+}$and $\mathbf{U}=\sum_{k=0}^{\infty} \eta_{+}^{* k}$. The 'matrix' $\boldsymbol{F}(\mathbb{R})$ is clearly irreducible and its spectral radius $\rho[\boldsymbol{F}(\mathbb{R})]=1$ (since $\eta_{+}$is a probability measure in this case). Also,

$$
\int_{-\infty}^{\infty} x \boldsymbol{F}(\mathrm{d} x)=\int_{-\infty}^{\infty} x \eta_{+}(\mathrm{d} x)=\mathbb{E}\left[\sum_{j=1}^{N} C_{j}^{\alpha} \log C_{j}\right]=\mu \in(0, \infty) .
$$

We now note that, by Fubini's theorem,

$$
\begin{aligned}
\int_{-\infty}^{0}(1+|x|)^{2} \varphi(x) \eta_{+}(\mathrm{d} x) & =\int_{-\infty}^{0}(1+|x|)^{2} \mathrm{e}^{\alpha x} \mathbb{E}\left[\sum_{i=1}^{N} \mathbf{1}\left(\log C_{i} \in \mathrm{d} x\right)\right] \\
& =\mathbb{E}\left[\sum_{i=1}^{N} \int_{0}^{\infty}(1+|x|)^{2} \mathrm{e}^{\alpha x} \mathbf{1}\left(\log C_{i} \in \mathrm{d} x\right)\right] \\
& =\mathbb{E}\left[\sum_{i=1}^{N} C_{i}^{\alpha}\left(1+\left|\log C_{i}\right|\right)^{2}\right]
\end{aligned}
$$

which is finite by assumption. This observation, by the remarks preceding Theorem 2 of [12], implies that $T^{2} \boldsymbol{F} \in S(\varphi)$, where, for any finite complex-valued measure $v, T v$ is defined as the $\sigma$-finite measure with density $v(x ; v) \triangleq v((x, \infty))$ for $x \geq 0$ and $v(x ; v) \triangleq-$ $v((-\infty, x])$ for $x<0$, and $S(\varphi)$ is the collection of all complex-valued measures $\kappa$ such that $\int_{-\infty}^{\infty} \varphi(x)|\kappa|(\mathrm{d} x)<\infty$, with $|\kappa|$ the total variation of $\kappa$.

Then, by Theorem 2 of [12],

$$
\left|\breve{r}(t)-\frac{1}{\mu} \int_{0}^{\infty} \breve{g}_{+}(x) \mathrm{d} x\right|=\left|\mathbf{U} * \breve{g}_{+}(t)-\frac{1}{\mu} \int_{0}^{\infty} \breve{g}_{+}(x) \mathrm{d} x\right|=o\left(\mathrm{e}^{-\theta t}\right) \quad \text { as } t \rightarrow \infty .
$$

To derive the result for $\mathbb{P}(R<-t)$, follow the same steps leading to (3.3) in the proof of Theorem 3.4 of [10] but starting with a telescoping sum for $\mathbb{P}\left(-R>\mathrm{e}^{t}\right)$ instead, and defining $r(t)=\mathrm{e}^{\alpha t} \mathbb{P}\left(R<-\mathrm{e}^{t}\right)$. Using the same arguments as above then gives

$$
\left|\breve{r}(t)-\frac{1}{\mu} \int_{0}^{\infty} \breve{g}_{-}(x) \mathrm{d} x\right|=\left|\mathbf{U} * \breve{g}_{-}(t)-\frac{1}{\mu} \int_{0}^{\infty} \breve{g}_{-}(x) \mathrm{d} x\right|=o\left(\mathrm{e}^{-\theta t}\right) \quad \text { as } t \rightarrow \infty .
$$

We have thus shown that

$$
\begin{array}{rl}
\mid \int_{-\infty}^{t} & b \mathrm{e}^{-b(t-s)} \mathrm{e}^{\alpha s} \mathbb{P}\left( \pm R>\mathrm{e}^{s}\right) \mathrm{d} s-\frac{1}{\mu} \int_{0}^{\infty} \int_{-\infty}^{x} b \mathrm{e}^{-b(x-s)} g_{ \pm}(s) \mathrm{d} s \mathrm{~d} x \mid \\
\quad=\left|\mathrm{e}^{-b t} \int_{0}^{\mathrm{e}^{t}} b v^{\alpha+b-1} \mathbb{P}( \pm R>v) \mathrm{d} v-\frac{1}{\mu} \int_{-\infty}^{\infty} g_{ \pm}(s) \mathrm{d} s\right| \\
\quad=\left|\mathrm{e}^{-b t} \int_{0}^{\mathrm{e}^{t}} b v^{\alpha+b-1} \mathbb{P}( \pm R>v) \mathrm{d} v-H_{ \pm}\right| \\
=o\left(\mathrm{e}^{-\theta t}\right) \quad \text { as } t \rightarrow \infty,
\end{array}
$$


where

$$
\begin{aligned}
H_{ \pm} & \triangleq \frac{1}{\mu} \int_{-\infty}^{\infty} g_{ \pm}(s) \mathrm{d} s \\
& =\frac{1}{\mu} \int_{-\infty}^{\infty} \mathrm{e}^{\alpha t}\left(\mathbb{P}\left( \pm R>\mathrm{e}^{t}\right)-\mathbb{E}\left[\sum_{j=1}^{N} \mathbf{1}\left(( \pm 1) C_{j} R>\mathrm{e}^{t}\right)\right]\right) \mathrm{d} t \\
& =\frac{1}{\mu} \int_{0}^{\infty} x^{\alpha-1}\left(\mathbb{P}( \pm R>x)-\mathbb{E}\left[\sum_{j=1}^{N} \mathbf{1}\left(( \pm 1) C_{j} R>x\right)\right]\right) \mathrm{d} x .
\end{aligned}
$$

Therefore, by Lemma 3.2, we obtain

$$
\left|t^{\alpha} \mathbb{P}( \pm R>t)-H_{ \pm}\right|=o\left(t^{-\theta}\right) \quad \text { as } t \rightarrow \infty .
$$

Case 2: $\mathbb{P}\left(C_{j}<0\right)>0$ for some $j \geq 1$. For this case, $\eta_{-}$is nonzero. Also, note that the matrix

$$
\boldsymbol{F}(\mathbb{R})=\left(\begin{array}{cc}
\mathbb{E}\left[\sum_{j=1}^{N}\left|C_{j}\right|^{\alpha} \mathbf{1}\left(X_{j}=1\right)\right] & \mathbb{E}\left[\sum_{j=1}^{N}\left|C_{j}\right|^{\alpha} \mathbf{1}\left(X_{j}=-1\right)\right] \\
\mathbb{E}\left[\sum_{j=1}^{N}\left|C_{j}\right|^{\alpha} \mathbf{1}\left(X_{j}=-1\right)\right] & \mathbb{E}\left[\sum_{j=1}^{N}\left|C_{j}\right|^{\alpha} \mathbf{1}\left(X_{j}=1\right)\right]
\end{array}\right) \triangleq\left(\begin{array}{cc}
q & 1-q \\
1-q & q
\end{array}\right)
$$

is irreducible and has eigenvalues $\{1,2 q-1\}$, and, therefore, spectral radius $\rho[\boldsymbol{F}(\mathbb{R})]=1$. Moreover, $(1,1)$ and $(1,1)^{\top}$ are left and right eigenvectors of $\boldsymbol{F}(\mathbb{R})$, respectively, corresponding to eigenvalue 1 , and, by assumption,

$$
\begin{aligned}
(1,1) \int_{-\infty}^{\infty} x \boldsymbol{F}(\mathrm{d} x)\left(\begin{array}{l}
1 \\
1
\end{array}\right) & =2\left(\int_{-\infty}^{\infty} x \eta_{+}(\mathrm{d} x)+\int_{-\infty}^{\infty} x \eta_{-}(\mathrm{d} x)\right) \\
& =2 \mathbb{E}\left[\sum_{j=1}^{N}\left|C_{j}\right|^{\alpha} \log \left|C_{j}\right|\right] \\
& =2 \mu \in(0, \infty) .
\end{aligned}
$$

Also, similarly as in the nonnegative case, we have

$$
\int_{-\infty}^{0}(1+|x|)^{2} \varphi(x) \eta_{ \pm}(\mathrm{d} x)=\mathbb{E}\left[\sum_{i=1}^{N}\left|C_{i}\right|^{\alpha}\left(1+|\log | C_{i}||\right)^{2} \mathbf{1}\left(X_{i}= \pm 1\right)\right]
$$

which is finite by assumption. From the remarks preceding Theorem 2 of [12], it follows that $T^{2} \boldsymbol{F} \in S(\varphi)$.

Then, by Theorem 2 of [12],

$$
\begin{aligned}
\left|\mathbf{U} * \breve{\boldsymbol{g}}(t)-\frac{(1,1)^{\top}(1,1)}{2 \mu} \int_{-\infty}^{\infty} \breve{\boldsymbol{g}}(x) \mathrm{d} x\right| & =\left|\mathbf{U} * \breve{\boldsymbol{g}}(t)-\frac{1}{2 \mu}\left(\begin{array}{l}
\int_{-\infty}^{\infty}\left(\breve{g}_{+}(u)+\breve{g}_{-}(u)\right) \mathrm{d} u \\
\int_{-\infty}^{\infty}\left(\breve{g}_{+}(u)+\breve{g}_{-}(u)\right) \mathrm{d} u
\end{array}\right)\right| \\
& =o\left(\mathrm{e}^{-\theta t}\right) \quad \text { as } t \rightarrow \infty
\end{aligned}
$$

Hence, it follows from $\breve{r}(t)=\mathbf{e U} * \breve{g}(t)$ that

$$
\begin{aligned}
\left|\breve{r}(t)-\frac{1}{2 \mu} \int_{-\infty}^{\infty}\left(\breve{g}_{+}(u)+\breve{g}_{-}(u)\right) \mathrm{d} u\right| & =\left|\mathrm{e}^{-b t} \int_{0}^{\mathrm{e}^{t}} b v^{\alpha+b-1} \mathbb{P}(R>v) \mathrm{d} v-\frac{1}{2}\left(H_{+}+H_{-}\right)\right| \\
& =o\left(\mathrm{e}^{-\theta t}\right) \text { as } t \rightarrow \infty .
\end{aligned}
$$


Let $H=\left(H_{+}+H_{-}\right) / 2$, then, by Lemma 3.2,

$$
\left|t^{\alpha} \mathbb{P}(R>t)-H\right|=o\left(t^{-\theta}\right) \quad \text { as } t \rightarrow \infty .
$$

To derive the result for $\mathbb{P}(R<-t)$ simply start by defining $r(t)=\mathrm{e}^{\alpha t} \mathbb{P}\left(-R>\mathrm{e}^{t}\right)$, which in this case leads to the same result as above, that is,

$$
\left|t^{\alpha} \mathbb{P}(R<-t)-H\right|=o\left(t^{-\theta}\right) \quad \text { as } t \rightarrow \infty .
$$

Finally, we note, by using the representations for $H_{+}$and $H_{-}$from case 1 , that

$$
\begin{aligned}
H= & \frac{1}{2 \mu} \int_{0}^{\infty} x^{\alpha-1}\left(\mathbb{P}(R>x)-\mathbb{E}\left[\sum_{j=1}^{N} \mathbf{1}\left(C_{j} R>x\right)\right]\right) \mathrm{d} x \\
& +\frac{1}{2 \mu} \int_{0}^{\infty} x^{\alpha-1}\left(\mathbb{P}(R<-x)-\mathbb{E}\left[\sum_{j=1}^{N} \mathbf{1}\left(C_{j} R<-x\right)\right]\right) \mathrm{d} x \\
= & \frac{1}{2 \mu} \int_{0}^{\infty} x^{\alpha-1}\left(\mathbb{P}(|R|>x)-\mathbb{E}\left[\sum_{j=1}^{N} \mathbf{1}\left(\left|C_{j} R\right|>x\right)\right]\right) \mathrm{d} x .
\end{aligned}
$$

\section{References}

[1] Aldous, D. J. and Bandyopadhyay, A. (2005). A survey of max-type recursive distributional equation. Ann. Appl. Prob. 15, 1047-1110.

[2] Alsmeyer, G. And Meiners, M. (2013). Fixed points of the smoothing transform: two-sided solutions. Prob. Theory Relat. Fields 155, 165-199.

[3] Alsmeyer, G. And Rösler, U. (2006). A stochastic fixed point equation related to weighted branching with deterministic weights. Electron. J. Prob. 11, 27-56.

[4] Alsmeyer, G., Biggins, J. D. And Meiners, M. (2012). The functional equation of the smoothing transform. Ann. Prob. 40, 2069-2105.

[5] Biggins, J. D. And Kyprianou, A. E. (1997). Seneta-Heyde norming in the branching random walk. Ann. Prob. 25, 337-360.

[6] Fill, J. A. And Janson, S. (2001). Approximating the limiting Quicksort distribution. Random Structures Algorithms 19, 376-406.

[7] Goldie, C. M. (1991). Implicit renewal theory and tails of solutions of random equations. Ann. Appl. Prob. 1, 126-166.

[8] Jelenković, P. R. and Olvera-Cravioto, M. (2010). Information ranking and power laws on trees. Adv. Appl. Prob. 42, 1057-1093.

[9] Jelenković, P. R. and Olvera-Cravioto, M. (2012). Implicit renewal theory and power tails on trees. $A d v$. Appl. Prob. 44, 528-561.

[10] Jelenković, P. R. and Olvera-Cravioto, M. (2012). Implicit renewal theorem for trees with general weights Stoch. Process. Appl. 122, 3209-3238.

[11] RöSler, U. ANd RüSCHENDORF, L. (2001). The contraction method for recursive algorithms. Algorithmica 29, 3-33.

[12] SgIbnev, M. S. (2003). Systems of renewal equations on the line. J. Math. Sci. Univ. Tokyo 10, $495-517$. 